\title{
Influence of ventilatory strategies on outcomes and length of hospital stay: assist control and synchronized intermittent mandatory ventilation modes
}

\author{
Diego Casali ${ }^{1}$ (1)
}

Received: 26 September 2020 / Accepted: 30 September 2020 / Published online: 19 October 2020

(c) Società Italiana di Medicina Interna (SIMI) 2020

I read with interest the article published by Thais Bruno de Godoi and colleagues in the current issue of "Internal and Emergency Medicine" [1]. This single-center, observational, and retrospective study performed on 345 adult ICU patients shows that the mortality, duration of mechanical ventilation, length of hospital stay, failed extubation, and need for tracheostomy are statistically similar between the synchronized intermittent mandatory ventilation with pressure support ventilation (SIMV + PSV) mode and the assist-control (A/C) mode. This study compares outcomes of patients ventilated with $\mathrm{A} / \mathrm{C}$ mode or intermittent mandatory ventilation (IMV) mode under nonweaning conditions, and it confirms the clinical observation that no mode of mechanical ventilation results in better outcomes and that any mode can be successfully used by a knowledgeable and skillful intensivist.

The concept of allowing the patient to breathe spontaneously between machine-cycled or mandatory breaths originated in 1955 with an unnamed ventilator designed by Engstrom [2, 3]. In 1971, Kirby and colleagues [4, 5] introduced IMV as a means of ventilator support of infants with respiratory distress syndrome. In 1973, Downs and colleagues [6] proposed IMV as a method to facilitate liberation from mechanical ventilation in adults by allowing the patient to take unhindered breaths while still receiving a background of controlled breaths. Proposed advantages include decreased sedative requirements, decreased mean intrathoracic pressure with less barotrauma, and less adverse hemodynamic consequences, improved intrapulmonary gas distribution, continued use of respiratory muscles, and faster liberation from mechanical ventilation.

\section{Diego Casali}

Diego.Casali@cshs.org

1 Cardiac Surgery Intensive Care Unit, Smidt Heart Institute, Cedars Sinai Medical Center, 8700 Beverly Boulevard, Los Angeles, CA 90048, USA
The SIMV mode is very similar to the A/C mode. In fact, when the patient is apneic or breathes at or below the set mandatory respiratory rate, the two modes are indistinguishable. Like A/C, SIMV provides the patient with an intensivist-selected mandatory breath rate, and during every calculated breath interval it delivers a single mandatory breath, which can be either time or patient triggered. The difference between the two modes only appears when the patient triggers additional spontaneous breaths. In the $\mathrm{A} / \mathrm{C}$ mode, spontaneous and mandatory breaths are the same, whereas in the SIMV mode, different breath types are always used. Similarly to A/C, mandatory breaths in the SIMV mode can be volume control, pressure control, or pressure-regulated volume control breaths. Spontaneous breaths, however, are always pressure support breaths.

As shown in the article published by Thais Bruno de Godoi and colleagues [1], either mode can be used successfully for assisted ventilation, but one mode may be better than the other in some circumstances.

For example, for patients with respiratory muscle fatigue, some intensivists believe that even though IMV respiratory rates provide for adequate ventilation, they may be set too low to allow for an adequate unloading of the respiratory muscles and that IMV does not provide the same degree of respiratory muscle rest as the $\mathrm{A} / \mathrm{C}$ mode because an adequate minute ventilation depends on the patient's ability to generate enough volume with each spontaneous breath, which increases work of breathing.

Another disadvantage of IMV is the inability to fully control the I:E ratio due to the variability in respiratory rate and the presence of spontaneous breaths, which can be problematic in patients with obstructive lung disease.

IMV was the most popular method of weaning for many years [7]. With IMV, the mandatory respiratory rate of the ventilator is reduced in a stepwise fashion by $1-3$ breaths per minute, and an arterial blood gas is obtained approximately $30 \mathrm{~min}$ after each respiratory rate change. Unfortunately, it 
has been proven that the titration of the number of ventilator-supported breaths according to the results of arterial blood gases does not provide any information regarding the patient's work of breathing, which may be increased [8]. Two prospective multicenter randomized clinical trials have shown that the use of SIMV is less effective than other techniques for the progressive withdrawal of mechanical ventilation. The first randomized trial comparing three different methods of weaning (T-piece, SIMV, and PSV) was published by Brochard and colleagues in 1994 [9]. The study conclusion was that the outcome of weaning is influenced by the modality chosen during this period. The weaning duration was shorter with PSV compared with SIMV or T-piece when pooled together. The second randomized trial was published by Esteban and colleagues in 1995 [10]. This was a randomized multicenter study comparing four different methods of weaning (IMV, PSV, intermittent multiple trials of spontaneous breathing, and once-daily trial of spontaneous breathing). It showed that once daily spontaneous breathing trial is twice as fast as PSV and three times more rapid than SIMV. It also proved that multiple trials of spontaneous breathing do not reduce the time of weaning when compared with a once daily trial. When analyzed together, these studies suggest that weaning is slower with SIMV, although SIMV + PSV was not studied, and that either PSV or a T-piece trial are the preferred methods for weaning. Therefore, when patients fail spontaneous breathing trials, pressure support ventilation (PSV) is the most often mode used nowadays to achieve liberation from mechanical ventilation.

IMV has a physiologic advantage over $\mathrm{A} / \mathrm{C}$ mode, however, when there is auto-PEEP (intrinsic PEEP): the patient's spontaneous breaths during IMV provide negative intrathoracic pressures that counteract the positive intrathoracic pressure generated by the auto-PEEP and by the mandatory breaths.

Some intensivists use IMV when they manage patients with severe acute respiratory alkalosis due to hyperventilation. Cirrhotic and pregnant patients, for example, display elevated respiratory rates on the $\mathrm{A} / \mathrm{C}$ mode because of their increased central nervous system (CNS) respiratory drive. A simple switch from A/C to IMV mode does not typically solve the issue unless the patient's respiratory muscles become fatigued during IMV, which is clearly not a desirable outcome. Similarly, the addition of dead space or the exclusion of the patient by increasing the triggering threshold should not be used, because both methods may worsen respiratory muscle fatigue. In this situation, it is more appropriate to pharmacologically decrease the patient's high CNS drive with anxiolytics, opioids, or even chemical paralysis.

Liberation from mechanical ventilation is less determined by the mode of mechanical ventilation than by the identification and correction of the medical barriers to weaning.
However, when compared with other modes, SIMV has nearly consistently performed the worst in clinical trials and is not recommended for weaning anymore.

Furthermore, several studies report a decline in the application of SIMV + PSV in acute respiratory failure [11, 12], except in lower acuity patients, in the postoperative setting and in trauma patients [13]. The percentage of ARDS patients managed with SIMV has declined from 22\% in 1996 to $3 \%$ in 2005 [12]. With the ever-increasing emphasis on early liberation from mechanical ventilation and with the increased availability of newer and more comfortable modes of mechanical ventilation for patients who do not tolerate the A/C mode, such as Mandatory Minute Volume, AdaptiveSupport Ventilation (ASV), I wonder whether SIMV should ever be routinely used anymore.

Based on the results of the study by Thais Bruno de Godoi and colleagues [1], I will not change my clinical practice of selecting the $\mathrm{A} / \mathrm{C}$ mode in critically ill, mechanically ventilated patients in the ICU, but I congratulate the authors for conducting another of a handful of studies [13-15] comparing outcomes of patients ventilated with $\mathrm{A} / \mathrm{C}$ mode or intermittent mandatory ventilation (IMV) mode under nonweaning conditions.

\section{Compliance with ethical standards}

Conflict of interest Diego Casali has no conflict of interest to disclose.

Statement of human and animal rights This article does not contain any studies with human participants or animals performed by any of the authors.

Informed consent For this type of study, formal consent is not required.

\section{References}

1. de Godoi TB, Marson FAL, Palamim CVC, Cannonieri-Nonose GC (2020) Influence of ventilatory strategies on outcomes and length of hospital stay: assist-control and synchronized intermittent mandatory ventilation modes. Intern Emerg Med. https://doi. org/10.1007/s11739-020-02444-7

2. Bjork VO, Engstrom CG (1955) The treatment of ventilatory insufficiency after pulmonary resection with tracheostomy and prolonged artificial ventilation. J Thorac Surg 30:356-357

3. Fairley HB (1980) Critique of intermittent mandatory ventilation. Int Anesthesiol Clin 18:179-189

4. Kirby RR, Robinson EJ, Shulz J et al (1971) A new pediatric volume ventilator. Anesth Analg 50:533-537

5. Kirby RR, Robinson EJ, Shulz J et al (1972) Continuous flow as an alternative to assisted or controlled ventilation in infants. Anesth Analg 51:871-875

6. Downs JB, Klein EF, Desautels D et al (1973) Intermittent mandatory ventilation: a new approach to weaning patients from mechanical ventilations. Chest 64:331-335 
7. Sassoon CS (2012) Intermittent mechanical ventilation. In: Tobin MJ (ed) Principles and practice of mechanical ventilation, 3rd edn. McGraw Hill, New York, NY, pp 17-98

8. Tobin MJ et al (2012) Ventilator failure, ventilator support, and ventilator weaning. Compr Physiol 2:1-51

9. Brochard et al (1994) Comparison of three methods of gradual withdrawal from ventilatory support during weaning from mechanical ventilation. Am J Respir Crit Care Med 150(4):896-903

10. Esteban et al (1995) A comparison of four methods of weaning patients from mechanical ventilation. Spanish lung failure collaborative group. N Engl J Med 332(6):345-350

11. Esteban A, Anzueto A, Alia I et al (2000) How is mechanical ventilation employed in the intensive care unit? An international utilization review. Am J Respir Crit Care Med 161:1450-1458

12. Checkley W, Brower R, Korpak A et al (2008) Effects of a clinical trial on mechanical ventilation practices in patients with acute lung injury. Am J Respir Crit Care Med 177:1215-1222
13. Ortiz G, Frutos-Vivar F, Ferguson ND et al (2010) Outcomes of patients ventilated with synchronized intermittent mandatory ventilation with pressure support: a comparative propensity score study. Chest 137:1265-1277

14. Luo J, Wang MY, Liang BM et al (2015) Initial synchronized intermittent mandatory ventilation versus assist/control ventilation in treatment of moderate acute respiratory distress syndrome: a prospective randomized controlled trial. J Thorac Dis 7(12):2262-2273

15. Robinson BR, Blakeman TC, Toth P, Hanseman DJ, Mueller E, Branson RD (2013) Patient-ventilator asynchrony in a traumatically injured population. Respir Care 58(11):1847-1855

Publisher's Note Springer Nature remains neutral with regard to jurisdictional claims in published maps and institutional affiliations. 